\title{
The Mathematical Model for the Tippe Top Inversion
}

\author{
R. Usubamatov $\mathbb{D},{ }^{1}$ M. Bergander $\mathbb{D},^{2}$ and S. Kapayeva $\mathbb{D}^{3}$ \\ ${ }^{1}$ Kyrgyz State Technical University, Bishkek, Kyrgyzstan \\ ${ }^{2}$ Magnetic Development, Inc., Madison, USA \\ ${ }^{3}$ Serikbayev East Kazakhstan Technical University, Kazakhstan \\ Correspondence should be addressed to R. Usubamatov; ryspek0701@yahoo.com
}

Received 24 February 2021; Revised 26 March 2021; Accepted 19 April 2021; Published 3 May 2021

Academic Editor: Ivan Giorgio

Copyright ( 2021 R. Usubamatov et al. This is an open access article distributed under the Creative Commons Attribution License, which permits unrestricted use, distribution, and reproduction in any medium, provided the original work is properly cited.

\begin{abstract}
The dynamics of rotating objects is an area of classical mechanics that has many unsolved problems. Among these problems are the gyroscopic effects manifested by the spinning objects of different forms. One of them is the Tippe top designed as the truncated sphere which is fitted with a short, cylindrical rod for rotation. The unexplainable gyroscopic effect of the Tippe top is manifested by its inversion towards the support surface. Researchers tried to describe this gyroscopic effect for two centuries, but all modelings were on the level of assumptions. It is natural because the Tippe top has a more complex design than the simple spinning disc, which gyroscopic effects did not have an analytical solution until recent time. The latest research, in the area of gyroscopic effects, reveals the action of the system of several interrelated inertial torques on any spinning object. The gyroscopic inertial torques are generated by their rotating mass. These inertial torques and the variable ratio of the angular velocities of the spinning object around axes of rotations constitute the fundamental principles of gyroscope theory. These physical principles of dynamics of rotating objects enable to description and compute of any gyroscopic effects and also the Tippe top inversion.
\end{abstract}

\section{Introduction}

The dynamics of classical mechanics contains the section of gyroscope theory that considers different types of spinning objects [1-3]. One of them is well-known from ancient times a spinning top and its modification the Tippe top. This top is designed with a truncated sphere that is fitted by a short, cylindrical rod for rotation. The Tippy top center mass is located below its center of the sphere. The gyroscopic effect of the spinning Tippe top is manifested when its spherical surface contacts the support surface and by the following inversion and spinning on the rod [4-6]. Following spinning of the Tippe top is the same as a regular design of the top. This gyroscopic effect of the spinning Tippe top manifests other designs of the rotating objects as hardboiled eggs, an American football, round smooth ovals, etc. [7-9]. All similar spinning objects with the property of inversion attracted physicists and mathematicians [10-12]. They have been studied extensively the gyroscopic effect of Tippe top from the beginning of the nineteenth century [13-15]. The inversion property of the top served as an amusing toy for a population that was more attractive than an ordinary disc-type top. The Tippe top for the short time got popularity all over the world.

Nevertheless, the number of publications dedicated to the physics of Tippe top is less than for a disc-type top. The complexity of the Tippe top's motions and the unexplainable gyroscopic effect of inversion interested only persistent researchers. Analysis of these publications and methods for solutions demonstrate the application of the same mathematical tools and more intuitive assumptions as for the spinning top [16]. Their main principles for describing the Tippe top inversion were the angular momentum, kinetic energy, friction force, and its weight. In reality, the physics of gyroscopic effects are many times harder. This is the reason that the Tippe top's gyroscopic effect does not have the accepted physics and recognized mathematical model.

In engineering, the visual example of the manifestation of the spinning object inversions is presented at conditions of the weightless at the orbital flight. This example and other nondiscovered ones are waiting for investigations of their physics. 
All similar examples with the spinning objects are formulating by the defined principles for gyroscopic effects that can explain and describe the physics of acting forces and inversion by the rules of classical mechanics [17].

This work proposes the analytical circumscribing of the physics for Tippe top's inversion based on the latest defined principles of gyroscope theory $[17,18]$.

\section{Methodology}

The mathematical model for motions of the Tippe top is formulated by the defined principles for gyroscopic effects that can explain and describe the physics of acting forces and inversion by the rules of classical mechanics [17]. The system of the inertial torques generated by the centrifugal, common inertial, Coriolis forces, the change in the angular momentum of the spinning object, and the ratio of the angular velocities of the top around axes are applied for the mathematical model for its motions. For the analysis, the top axis is located vertically at the movable system of $3 \mathrm{D}$ coordinates $\Sigma o x y z$ which center disposes at the center mass of the Tippe top. The location of the center mass $m$ of the top is below the center of the truncated sphere on the distance $r$. The top is spinning with the angular velocity $\omega$ in the counterclockwise direction around axis oy that passed the center mass. The combined action of external, inertial torques, forces, and motions around three axes for the running inclined Tippe top as the truncated sphere with the fitted rod is represented in Figure 1.

At starting condition, the top spins on the spherical surface of the radius $R$ and contacts the support surface at the point $P$. The spinning axis of the top passes the center mass $m$ and disposes perpendicular to the support surface. Any inclination of the top on the angle $\beta$ or the roughness of the contact surfaces gives the shift of the point $P$ of the top to the side. At this situation, the spinning axis passes the center mass and does not pass the center of the sphere and the contact point $P$. The size of the shift of the contact point $P$ depends on the displacement $r$ of the center mass from the center of the sphere (Figure 1). At this condition, the sphere slides on the support surface.

The point $P$ moves by the circular line around the axis $o z$ of the top's rotation. The sliding motion is fulfilled at the condition of the action of the kinetic frictional force $F=m g f$ ( $g$ is the gravity acceleration; $f$ is the coefficient of dry sliding kinetic friction). The frictional force $F$ acts around two axes $o x$ and $o z$ and produces the frictional torque $T_{f x}=m g f h$ $\cos \beta$ ( $h=R-r \sin \beta$ is the distance from the center mass to the support surface) and $T_{f y}=m g f r \cos \beta$, respectively.

The frictional torque $T_{f x}$ turns the angular momentum vector $H$ of the spinning top on the small-angle $\gamma_{x}, H_{x}$ around axis $o x$ at the plane yoz in the counterclockwise direction (Figure 1). The vector of the change in the angular momentum $\Delta H x$ presents the precession torque that turns the angular momentum vector $H$ of the spinning top on the small-angle $\gamma_{y}$ around axis $o y$ at the plane $x o z$ in the counterclockwise direction.

The action of the external torque on the spinning object generates the system of interrelated inertial torques that depends on the ratio of the angular velocities of its rotation around axes and is well described in the publication [17, 18]. The dependency of angular velocities of the rotation around axes of the spinning sphere is defined by the principle of the kinetic energy conservation law [17]. Schematic of the torques acting on the Tippe top (Figure 1) is used for the formulation of the equation of the kinetic energy balance for the inertial torques acting around axes of rotation. These inertial torques express the equality of the kinetic energy of the spinning objects (Chapter 4, Section 4.1.2, of [17]):

$$
\begin{aligned}
& -\left[\frac{3}{8}(\pi-2)^{2} \pi+\frac{3}{8}(\pi-2)\right] J \omega \omega_{x} \\
& -\left(\frac{3}{8}(\pi-2)^{2} \pi+1\right) J \omega \omega_{y}=\left(\frac{3}{8}(\pi-2)^{2} \pi+1\right) J \omega \omega_{x} \\
& -\left[\frac{3}{8}(\pi-2)^{2} \pi+\frac{3}{8}(\pi-2)\right] J \omega \omega_{y} .
\end{aligned}
$$

Simplification of Equation (1) yields the dependency of angular velocities of the rotation around axes for the spinning sphere:

$$
\omega_{y}=-\left[\frac{6(\pi-2)^{2} \pi+3(\pi-2)+8}{8-3(\pi-2)}\right] \omega_{x}
$$

where the sign (-) shows the direction of the action of inertial torques that can be omitted for the following considerations (Figure 1).

The mathematical models for the expressions of the inertial torques for the spinning sphere are described in the work [18], and the dependency of angular velocities of the rotation around axes (Equation (2)) is presented in Table 1.

$J$ is the variable mass moment of inertia of the Tippe top around the axis of rotation; $T_{i}$ is the variable inertial torques that depend on the geometry of the top and its axis inclination on the angle $\beta ; \omega$ is the angular velocity of the spinning top; and $\omega_{i}$ is the angular velocity of the rotation of the top around axis $i$.

The action of the inertial torques and the motions of the spinning Tippe top around axes are presented by the following components of gyroscopic effects (Figure 1):

(i) The frictional torque $T_{f x}$ which action is the counterclockwise direction around axis ox produces the resistance torque $T_{r x}=-T_{c t x}-T_{c r x}-T_{i n y}-T_{a m y}-T_{r l x}$, where $T_{r l x}=m g f_{r} R \cos \beta$ is the rolling frictional torque, and other torques are as specified in Table 1 . The resulting torque around axis $o x$ is the sum of frictional and resistance torques $T_{x}=T_{f x}-T_{r x}$ turns the spinning top with the angular velocity $\omega_{x}$

(ii) The torque $T_{x}$ produces the resulting precession torque around axis oy $T_{y}=T_{i n x}+T_{a m x}-T_{c t y}-T_{c r y}-T_{r l y}-T_{m g y}$, where $T_{r l y}=m g f r R \cos \beta$ is the rolling frictional torque, $T_{m g y}=m g r \cos \beta$ is the torque produced by the top weight due to the inclination of an axis on the angle $\beta$, and other torques are as specified in Table 1 


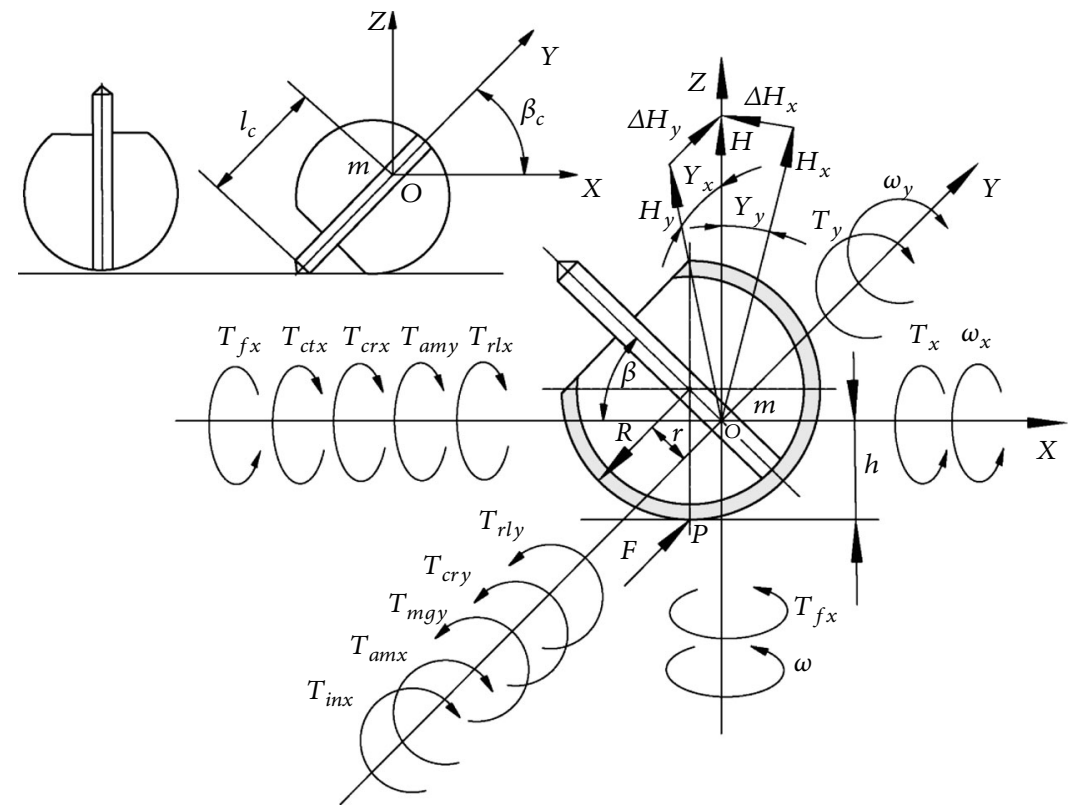

FIGURE 1: Schematic of the torques acting on the Tippe top spinning on the sphere and its motions.

TABle 1: Equations of the inertial torques acting on the spinning Tippe top.

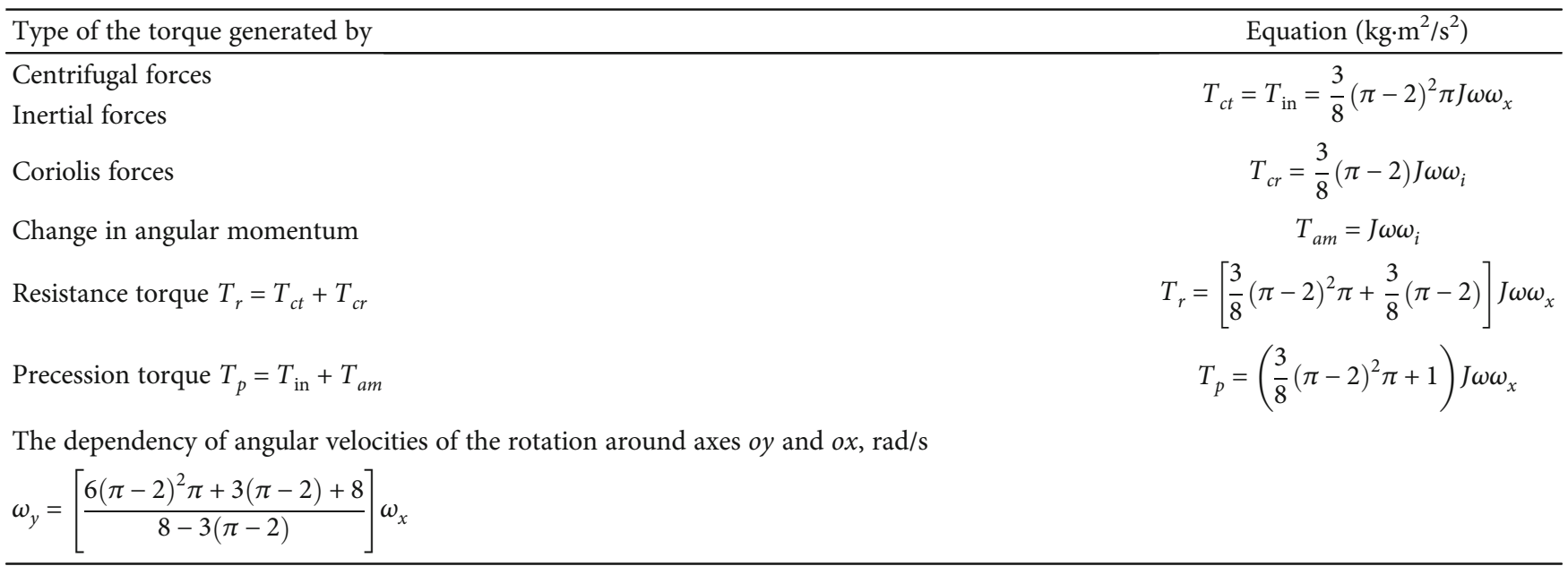

(iii) The inertial torques $T_{c t x}$ and $T_{i n x}$ at expressions of $T_{x}$ and $T_{y}$ are self-compensated as far as there is one expression and one sign (Table 1) (Chapter 4, Section 4.1 .2 of [17])

(iv) The action of the inertial torques on the spinning top leads to its rotation around axes $o x$ and $o y$ with the interrelated angular velocities $\omega_{x}$ and $\omega_{y}$, respectively. The following turn of the spinning top around two axes brings to contact of its rod with a support surface, inversion, and its vertical spinning on the rod. The light model of the top with a big diameter of the rod and high coefficient of sliding friction can change in the direction of spinning. This condition occurs at the time of the contact of the rod with the support surface. The change in the direction of the top spinning is explained by the principle of the kinetic energy conservation law and by the principle of the angular impulse and momentum

(v) The spinning top maintains its vertical rotation in so far as the point $P$ of its contact with the support surface that is movable because the top rolls on the spherical surface under the action of the inertial 
torques. This rolling leads to the turn of the top's axis and to change the mass moment of the inertia of the Tippe top around axes of rotation. The top's moment inertia is different for its vertical and horizontal location due to its nonsymmetrical design relative to its axis of spinning

(vi) The action of the inertial torques is interrelated and looped by the ratio of the angular velocities of the top around axes of rotation. The action of the frictional force $F$ activates all inertial torques generated by the rotating mass of the top

This detailed presentation of the action of frictional and inertial torques on the Tippe top enables us to describe and explain the physics of its motions and inversion. Analysis of the action of the inertial torques on the spinning top demonstrates its inversion is the result of the action of two precession torques around two axes. A mathematical model for the Tippe top motions is formulated by the equations of the inertial torques and the ratio of the angular velocities of the top around of axes of rotation (Table 1). The physics of inversion is considered for two conditions of the Tippe top spinning, i.e., on the spherical part and on the rod.

The mathematical models for the Tippe top motions around two axes are expressed by the following Euler's differential equations (Chapter 6, Section 6.2 of [17]):

$$
\begin{gathered}
J_{x} \frac{d \omega_{x}}{d t}=T_{f y}-T_{r l x}-T_{c t x}-T_{c r x}-T_{a m . y} \eta, \\
J_{y} \frac{d \omega_{y}}{d t}=T_{i n x}+T_{a m x}-T_{m g y}-T_{c r y}-T_{r l y}, \\
\omega_{y}=\left[\frac{6(\pi-2)^{2} \pi+3(\pi-2)+8}{8-3(\pi-2)}\right] \omega_{x},
\end{gathered}
$$

where $\omega_{x}$ and $\omega_{z}$ are the angular velocity of the top around axes $o x$ and $o z$, respectively; $t$ is the time; $T_{c t x}$, $T_{c r x}, T_{c r y}, T_{i n y}, T_{a m x}$, and $T_{a m y}$ are the variable inertial torques generated by the centrifugal, Coriolis, inertial forces and the change in the angular momentum acting around axes $o x$ and $o y$, respectively (Table 1$) ; \eta$ is the coefficient of the change in the inertial torque due to the action of the frictional torques on the top; $J_{y}$ and $J_{x}$ are the mass moments of inertia of the top around axes oy and $o x$, respectively; other components are as specified above.

The gyroscope parameters of Equations (3)-(5) and their mathematical processing were described in the publication (Chapter 6, Section 6.2 of [17]). The frictional torque acting around axis oy on the spinning top (Figure 1) reduces the value of the precession torques acting around axis $o x$ that is presented by the correction coefficient $\eta$. The coefficient $\eta$ is expressed by the ratio of the difference between the precession torques $\left(T_{i n x}+T_{a m x}\right)$ and the external torques $T_{r l y}$ and $T_{m g y}$ of axis $o x$, to the precession torques. Substituting equations of Table 1 into the ratio of these torques and transformation yields the expression of the correction coefficient $\eta$.

$$
\begin{aligned}
\eta & =\frac{T_{i n x}+T_{a m x}-T_{r l y}-T_{m g y}}{T_{i n x}+T_{a m x}}=1-\frac{T_{r l y}+T_{m g y}}{T_{\text {in. } x}+T_{a m . x}} \\
& =1-\frac{\left(m g f_{r} R+m g r\right) \cos \beta}{\left[(3 / 8)(\pi-2)^{2} \pi+1\right] J \omega \omega_{x}},
\end{aligned}
$$

where $J$ is the mass moment of inertia; $f_{r}$ is the rolling frictional coefficient; $\beta$ is the angle of the top inclination, $\omega$ is the angular velocity of the spinning top, and other components are as specified above.

For the solution of Equation (4), the precession torques $T_{p x}=T_{\text {in. } x}+T_{a m . x}$ (Table 1 ) should be expressed via the frictional load torque $T_{f y}$ and the angular velocity $\omega_{x}$ by $\omega_{x}$. The value of the precession torque of Equation (4) should be corrected by the principle of the conservation of the kinetic energy. Since the angular velocity of the gyroscope around axis $o y$ is bigger than around axis ox (Equation (5)), then the value of the precession torque should be proportionally increased on the ratio of the angular velocities (Equation (3)) and decreased on the ratio $n$ of the precession and resistance torques. This transformation expresses the interdependency of load and inertial torques acting around two axes of the spinning object (Chapter 5, Section 5.2, of [17]). The expression of $\omega_{x}$ is replaced by $\omega_{y}$ (Equation (5)) into the precession torque of Equation (4) and increased on the ratio of the angular velocities; then, the ratio of the precession and resistance torques is represented by the following:

$$
\begin{aligned}
n & =\frac{\left[\left(6(\pi-2)^{2} \pi+3(\pi-2)+8\right) /(8-3(\pi-2))\right]\left[(3 / 8)(\pi-2)^{2} \pi+1\right] J \omega \omega_{y} /\left[\left(6(\pi-2)^{2} \pi+3(\pi-2)+8\right) /(8-3(\pi-2))\right]}{(3 / 8)(\pi-2) J \omega \omega_{y}} \\
& =(\pi-2) \pi+\frac{8}{3(\pi-2)}
\end{aligned}
$$


The expression of the angular velocity $\omega_{x}$ is defined from the right side of Equation (1). Substituting defined parameters (Table 1, Equation (6)) into the right side of Equation (4)), and transformation yields the following:

$m g f \cos \beta(R-r \sin \beta)-\left[\frac{3}{8}(\pi-2)^{2} \pi+\frac{3}{8}(\pi-2)\right] J \omega \omega_{x}$

$$
\begin{gathered}
-\left[\frac{6(\pi-2)^{2} \pi+3(\pi-2)+8}{8-3(\pi-2)}\right] \\
\cdot\left[1-\frac{\left(m g f_{r} R+m g r\right) \cos \beta}{\left[(3 / 8)(\pi-2)^{2} \pi+1\right] J \omega \omega_{x}}\right] J \omega \omega_{x}=0 .
\end{gathered}
$$

Simplification of Equation (8) brings the following:

$$
\omega_{x}=\left\{\frac{f \cos \beta(R-r \sin \beta)+\left[\left(\left(6(\pi-2)^{2} \pi+3(\pi-2)+8\right) /(8-3(\pi-2))\right) /\left((3 / 8)(\pi-2)^{2} \pi+1\right)\right]\left(f_{r} R+r\right) \cos \beta}{(3 / 8)(\pi-2)^{2} \pi+(3 / 8)(\pi-2)+\left(6(\pi-2)^{2} \pi+3(\pi-2)+8\right) /(8-3(\pi-2))}\right\} \frac{m g}{J \omega} .
$$

Then, the modified formula of the precession torque $T_{p . x}$ is presented by the following expression:

$$
\begin{aligned}
T_{p}= & {\left[\left((3 / 8)(\pi-2)^{2} \pi+1\right) /((\pi-2) \pi+(8 /(3(\pi-2))))\right] J \omega } \\
& \left\{\frac{f \cos \beta(R-r \sin \beta)+\left[\left(\left(6(\pi-2)^{2} \pi+3(\pi-2)+8\right) /(8-3(\pi-2))\right) /\left((3 / 8)(\pi-2)^{2} \pi+1\right)\right]\left(f_{r} R+r\right) \cos \beta}{(3 / 8)(\pi-2)^{2} \pi+(3 / 8)(\pi-2)+\left(\left(6(\pi-2)^{2} 2 \pi+3(\pi-2)+8\right) /(8-3(\pi-2))\right)}\right\} \frac{m g}{J \omega} \\
& =\left\{\frac{\left[(3 / 8)(\pi-2)^{2} \pi+1\right] f \cos \beta(R-r \sin \beta)+\left[\left(6(\pi-2)^{2} \pi+3(\pi-2)+8\right) /(8-3(\pi-2))\right]\left(f_{r} R+r\right) \cos \beta}{[(\pi-2) \pi+(8 /(3(\pi-2)))]\left[(3 / 8)(\pi-2)^{2} \pi+(3 / 8)(\pi-2)+\left(\left(6(\pi-2)^{2} \pi+3(\pi-2)+8\right) /(8-3(\pi-2))\right)\right]}\right\} m g .
\end{aligned}
$$

Substituting Equations (6) and (5) into Equation (3) and Equations (7) and (10) into Equation (4) yields the following equations:

$$
\begin{aligned}
& J_{x} \frac{d \omega_{x}}{d t}=m g f \cos \beta(R-r \sin \beta)-\left[\frac{3}{8}(\pi-2)^{2} \pi+\frac{3}{8}(\pi-2)\right] J \omega \omega_{x}-m g f_{r} R \cos \beta-\left[\frac{6(\pi-2)^{2} \pi+3(\pi-2)+8}{8-3(\pi-2)}\right]\left[1-\frac{\left(m g f_{r} R+m g r\right) \cos \beta}{\left[(3 / 8)(\pi-2)^{2} \pi+1\right] J \omega \omega_{x}}\right] J \omega \omega_{x}, \\
& J_{y} \frac{d \omega_{y}}{d t}=\left\{\frac{\left[(3 / 8)(\pi-2)^{2} \pi+1\right] f \cos \beta(R-r \sin \beta)+\left(\left(6(\pi-2)^{2} \pi+3(\pi-2)+8\right) /(8-3(\pi-2))\right)\left(f_{r} R+r\right) \cos \beta}{[(\pi-2) \pi+(8 /(3(\pi-2)))]\left[(3 / 8)(\pi-2)^{2} \pi+(3 / 8)(\pi-2)+\left(\left(6(\pi-2)^{2} \pi+3(\pi-2)+8\right) /(8-3(\pi-2))\right)\right]}\right\} m g-\frac{3}{8}(\pi-2) J \omega \omega_{y}-m g f_{r} R \cos \beta-m g r \cos \beta,
\end{aligned}
$$

where the components of the external resistance torques $T_{r y}=m g f_{r} R \cos \beta+m g r \cos \beta$ are removed from Equation (12) as far as Equation (9) contains the correction factor $\eta$; all other components are as specified above.
Simplifications and transformations of Equations (11) and (12) bring the following:

$$
\begin{gathered}
J_{x} \frac{d \omega_{x}}{d t}=\left[f \cos \beta(R-r \sin \beta)+\frac{\left(f_{r} R+r\right) \cos \beta}{(3 / 8)(\pi-2)^{2} \pi+1}-f_{r} R\right] m g-\left[\frac{3}{8}(\pi-2)^{2} \pi+\frac{3}{8}(\pi-2)+\frac{6(\pi-2)^{2} \pi+3(\pi-2)+8}{8-3(\pi-2)}\right] J \omega \omega_{x}, \\
J_{y} \frac{d \omega_{y}}{d t}=\left\{\frac{\left[(3 / 8)(\pi-2)^{2} \pi+1\right] f \cos \beta(R-r \sin \beta)+\left(\left(6(\pi-2)^{2} \pi+3(\pi-2)+8\right) /(8-3(\pi-2))\right)\left(f_{r} R+r\right) \cos \beta}{[(\pi-2) \pi+(8 /(3(\pi-2)))]\left[(3 / 8)(\pi-2)^{2} \pi+(3 / 8)(\pi-2)+\left(\left(6(\pi-2)^{2} \pi+3(\pi-2)+8\right) /(8-3(\pi-2))\right)\right]}\right\} m g-\frac{3}{8}(\pi-2) J \omega \omega_{y} .
\end{gathered}
$$




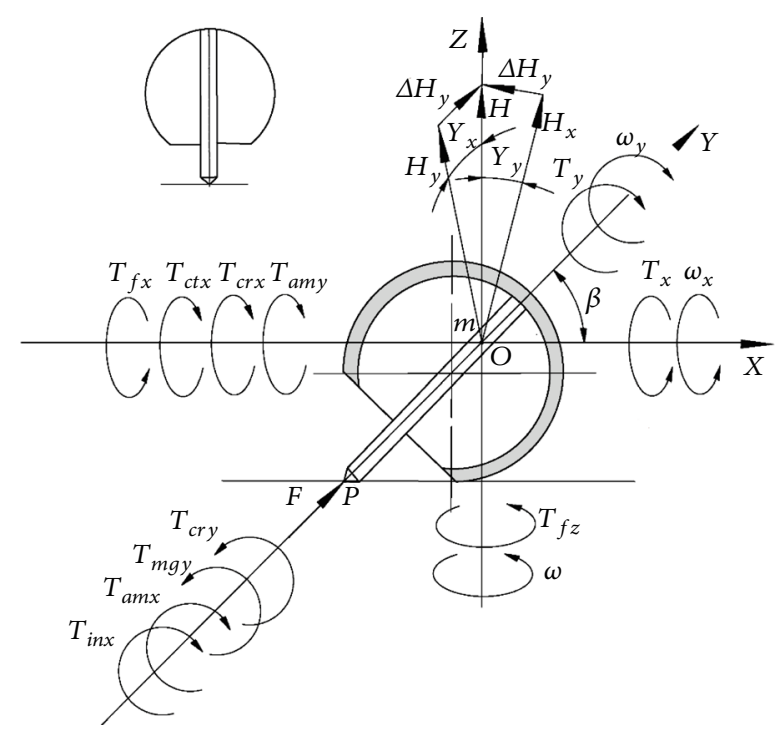

FIGURE 2: Schematic of the torques acting on the Tippe top spinning on the rod and its motions.

Equations (13) and (14) are used for direct computing of the angular velocities $\omega_{x}$ and $\omega_{y}$ of the Tippe top around axes $o x$ and $o y$, respectively. The value of the mass moments of inertia $J$ and $J_{i}$ of the top around axes $i$ of rotation is a variable that depends on the angle $\beta$ of the top inclination. The action of the resulting torque around axis ox and oy turns the top until the contact of the rod with the support surface. The following turn of the top disconnects the spherical part with the support surface and the top starts to rotate on the rod. The top rotates around the center mass, and its rod moves by the spiral trajectory on the support surface. The action of the frictional torque $F$ on the rod and following inertial torques generated is almost the same as in Figure 1. The rolling friction force does not consider due to the small diameter of the tip of the rod. The torques acting on the top spinning around the vertical axis $o z$ and its motion are presented in Figure 2.

The method and processing of the mathematical models for the Tippe top rotation on the rod around axes are the same as for Equations (3)-(5) and presented by the following equations:

$$
\begin{aligned}
J_{x} \frac{d \omega_{x}}{d t}= & m g f l_{c} \cos \beta \sin \beta-\left[\frac{3}{8}(\pi-2)^{2} \pi+\frac{3}{8}(\pi-2)\right] J \omega \omega_{x} \\
& -J \omega \omega_{y} \eta,
\end{aligned}
$$

$$
\begin{aligned}
J_{y} \frac{d \omega_{y}}{d t}= & \left(\frac{3}{8}(\pi-2)^{2} \pi+1\right) J \omega \omega_{x}-\frac{3}{8}(\pi-2) J \omega \omega_{y} \\
& -m g l_{c} \cos \beta,
\end{aligned}
$$

where the spinning of the top in the same direction, $J_{i}$, is the top's mass moment of inertia around the support ${ }^{3}$; $T_{m g f}=m g l_{c} \cos \beta \sin \beta$ is the frictional torque (for the top rotating on the rod); $T_{m g y}=m g l_{c} \cos \beta$ is the torque generated by the action of the top weight; $l_{c}$ is the length of the leg; other expressions are as specified in Equations (3) and (4).

The correction coefficient $\eta$ is defined similarity as for Equation (6) and presented by the following expression:

$$
\eta=1-\frac{m g l_{c} \cos \beta}{\left[(3 / 8)(\pi-2)^{2} \pi+1\right] J \omega \omega_{x}} .
$$

The precession torques $T_{p . x}=T_{\text {in. } x}+T_{a m . x}$ of Equation (16) is expressed via the load torque $T$ and the angular velocity $\omega_{x}$ by $\omega_{x}$. The solution is the same as presented for Equation (4). The ratio of the precession and resistance torques is represented by Equation (7). The expression of the angular velocity $\omega_{x}$ is defined from the right side of Equation (15). Substituting defined parameters (Table 1, Equation (17)) into the right side of Equation (15)) and transformation yields the following:

$$
\begin{aligned}
m g f l_{c} \cos \beta \sin \beta & -\left[\frac{3}{8}(\pi-2)^{2} \pi+\frac{3}{8}(\pi-2)\right] J \omega \omega_{x} \\
& -\left[\frac{6(\pi-2)^{2} \pi+3(\pi-2)+8}{8-3(\pi-2)}\right] \\
& {\left[1-\frac{m g l_{c} \cos \beta}{\left[(3 / 8)(\pi-2)^{2} \pi+1\right] J \omega \omega_{x}}\right] J \omega \omega_{x}=0 . }
\end{aligned}
$$

Simplification of Equation (18) brings the following:

$$
\omega_{x}=\left[\frac{f l_{c} \cos \beta \sin \beta+\left(\left(l_{c} \cos \beta\right) /\left((3 / 8)(\pi-2)^{2} \pi+1\right)\right)}{(3 / 8)(\pi-2)^{2} \pi+(3 / 8)(\pi-2)+\left(\left(6(\pi-2)^{2} \pi+3(\pi-2)+8\right) /(8-3(\pi-2))\right)}\right] \frac{m g}{J \omega} .
$$

Then, the modified expression of the precession torque $\left(T_{p . x}=T_{\text {in. } x}+T_{a m . x}\right)$ is defined by the same method as for Equation (10) and presented by the following expression:

$$
T_{p . x}=\left\{\frac{\left[(3 / 8)(\pi-2)^{2} \pi+1\right](f \sin \beta+1) l_{c} \cos \beta}{[(\pi-2) \pi+(8 /(3(\pi-2)))]\left[(3 / 8)(\pi-2)^{2} \pi+(3 / 8)(\pi-2)+\left(\left(6(\pi-2)^{2} \pi+3(\pi-2)+8\right) /(8-3(\pi-2))\right)\right]}\right\} m g .
$$


TABLe 2: Technical data of the Tippe top.

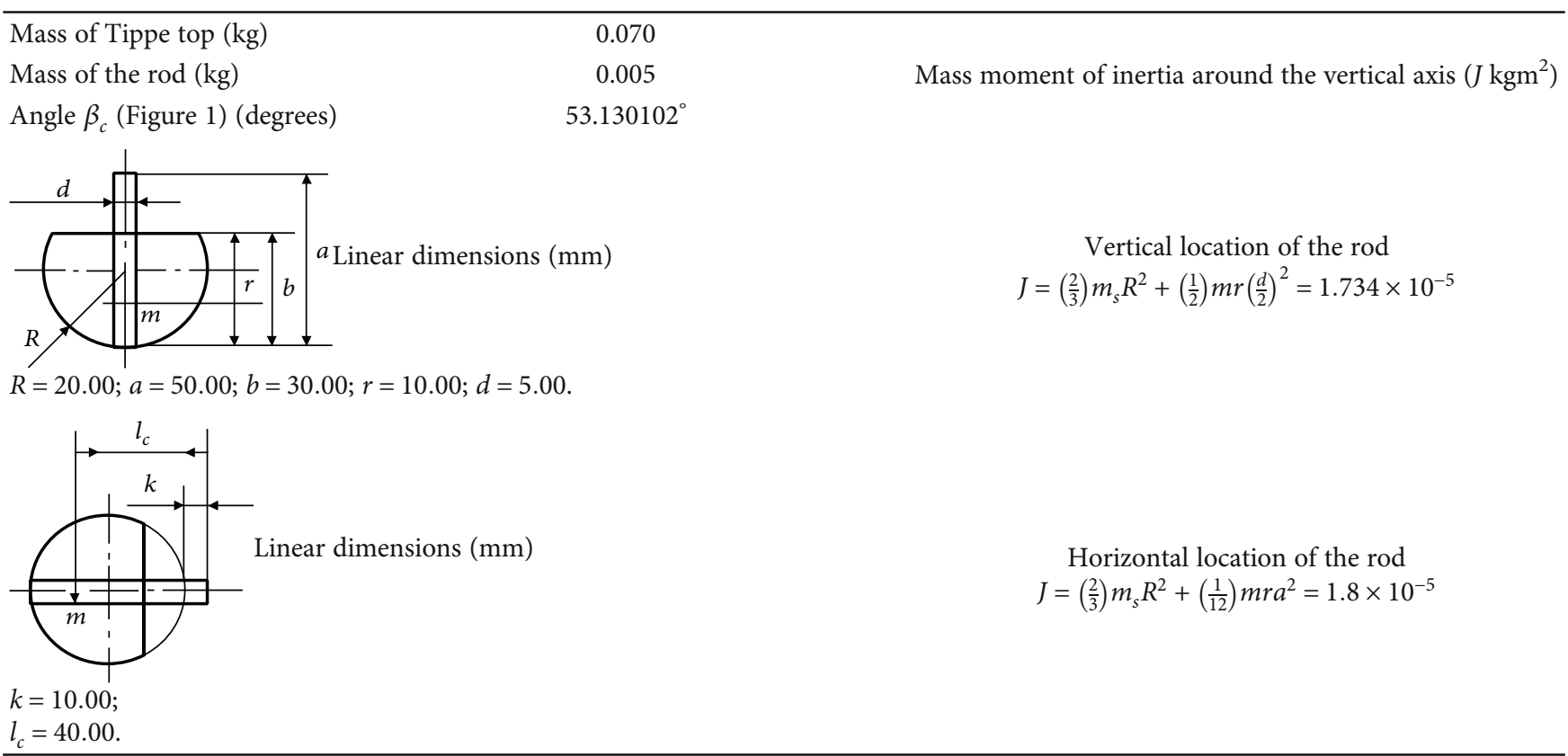

Substituting Equation (19) and $\eta$ into Equation (15) and Equation (19) into Equation (16) and transformation yields the following equations:

$$
\begin{array}{r}
J_{x} \frac{d \omega_{x}}{d t}=\left[f l_{c} \cos \beta \sin \beta+\frac{l_{c} \cos \beta}{(3 / 8)(\pi-2)^{2} \pi+1}\right] m g-\left[\frac{3}{8}(\pi-2)^{2} \pi+\frac{3}{8}(\pi-2)+\frac{6(\pi-2)^{2} \pi+3(\pi-2)+8}{8-3(\pi-2)}\right] J \omega \omega_{x}, \quad(21) \\
J_{y} \frac{d \omega_{y}}{d t}=-\left\{\frac{\left[(3 / 8)(\pi-2)^{2} \pi+1\right](f \sin \beta+1) l_{c} \cos \beta}{[(\pi-2) \pi+(8 / 3(\pi-2))]\left[(3 / 8)(\pi-2)^{2} \pi+(3 / 8)(\pi-2)+\left(\left(6(\pi-2)^{2} \pi+3(\pi-2)+8\right) /(8-3(\pi-2))\right)\right]}\right\} m g-\frac{3}{8}(\pi-2) J \omega \omega_{y},
\end{array}
$$

where all components are as specified above.

Equation (22) does not contain torques $T_{f y}=m g l_{c} \cos \beta$ as far as the precession torque modified with the correction factor $\eta$ contains this torque.

\section{Case Study and Working Example}

Analysis of the mathematical model for motions of the steel Tippe top on the steel support surface with the action of the frictional and inertial torques demonstrates the turn of its rod's axle from the vertical around axes $o x$ and $o y$. This top's turn is considered for the two conditions. The first condition is the top spins on its spherical part and turns until the contact of the rod with the support surface. The second condition is the rod of the spinning top contacts the support sur- face, its spherical part disconnects from it, the top inverses, and it turns to vertical. The technical data of the Tippe top is presented in Table 2. The starting condition accepted the vertical location of the top axle. The coefficients of the dry kinetic sliding and rolling friction of the top and table surfaces are accepted at $f=0.42$ and $f_{r}=0.001$, respectively [19]. The angular velocity of the spinning top is accepted at $2000 \mathrm{rpm}$. The angle of the contact of the rod with the table is defined from the geometry of the top that is $\beta_{c}=$ $53.130102^{\circ}$ (Figure 1). For simplicity of computing accepted, the mass moment of inertia for the sphere does not change the property of the top for inversion.

3.1. The Numerical Solution of the Case Study. The axis of the spinning of the Tippe top remains vertical, but the rod 
inclines then contacts the support surface, inverses, and then rotates on the rod. The rotation of the top's about horizontal changes in the values of the mass moment of inertia $J, J_{i}$ and inertial torques $T_{i}$. For simplicity of the solution and to avoid cumbersome computing, the values of $J$ and $J_{i}$ for the intermediate inclination of the top on the angle $\beta$ are accepted nearest to the vertical or horizontal location. This simplification does not bring a big difference in the results of computing because the values of $J$ and $J_{i}$ for vertical and horizontal locations are almost the same (Table 2).
(1) The spinning Tippe top motions on the spherical surface around vertical axis oy (Figure 1). The angular velocity of the top around axes of rotation is computed for the angle of the rod contact with the support surface. The turn of the top from vertical to the angle of the contact of the rod with the surface is $\beta$ $=143.130102^{\circ}$. The top parameters defined above and presented in Table 2 are substituted into Equations (13) and (14) that yield the following equations of the top motion around axis oy and $o x$

$1.734 \times 10^{-5} \frac{d \omega_{x}}{d t}=\left[\begin{array}{c}0.42 \cos \left(-53.130102^{\circ}\right) \times\left\{0.02-0.01 \times \sin \left(-53.130102^{\circ}\right)\right\}- \\ 0.001 \times 0.02\left(-53,130102^{\circ}\right)+ \\ \frac{(0.001 \times 0.02+0.01)\left(-53,130102^{\circ}\right)}{(3 / 8)(\pi-2)^{2} \pi+1}\end{array}\right] \times 0.07 \times 9.81-\left[\frac{3}{8}(\pi-2)^{2} \pi+\frac{3}{8}(\pi-2)+\frac{6(\pi-2)^{2} \pi+3(\pi-2)+8}{8-3(\pi-2)}\right] \times 1.734 \times 10^{-5} \times 2000 \times\left(\frac{2 \pi}{60}\right) \times \omega_{x}$,
$1.734 \times 10^{-5} \frac{d \omega_{y}}{d t}=\left\{\begin{array}{c}{\left[(3 / 8)(\pi-2)^{2} \pi+1\right] \times 0.42 \times \cos \left(-53.130102^{\circ}\right) \times\left(0.02-0.01 \times \sin \left(-53.130102^{\circ}\right)+\right.} \\ {\left[\left(6(\pi-2)^{2} \pi+3(\pi-2)+8\right) /(8-3(\pi-2))\right](0.001 \times 0.02+0.01) \cos \left(-53.130102^{\circ}\right)} \\ \frac{[(\pi-2) \pi+(8 /(3(\pi-2)))]\left[(3 / 8)(\pi-2)^{2} \pi+(3 / 8)(\pi-2)+\left(\left(6(\pi-2)^{2} \pi+3(\pi-2)+8\right) /(8-3(\pi-2))\right)\right]}{6}\end{array}\right\} \times 0.05 \times 9.81-\frac{3}{8}(\pi-2) \times 1.734 \times 10^{-5} \times 2000 \times\left(\frac{2 \pi}{60}\right) \times \omega_{y}$.

Following simplification and transformation yield the equations as follows:

$$
\begin{gathered}
1.734 \times 10^{-5} \frac{d \omega_{x}}{d t}=0.006465-0.035698 \omega_{x}, \\
1.734 \times 10^{-5} \frac{d \omega_{y}}{d t}=7.688728 \times 10^{-4}-0.0015546 \omega_{y} .
\end{gathered}
$$

Separating variables and transformation for these differential equations gives the following:

$$
\begin{gathered}
\frac{d \omega_{x}}{0.181102-\omega_{x}}=2058.7082 d t, \\
\frac{d \omega_{y}}{0.494579-\omega_{y}}=89.653979 d t .
\end{gathered}
$$

Transformation and presentation of the obtained equations by the integral form with defined limits yield the following:

$$
\begin{array}{r}
\int_{0}^{\omega_{x}} \frac{1}{0.181102-\omega_{x}} d \omega_{x}=2058.7082 \int_{0}^{t} d t, \\
\int_{0}^{\omega_{y}} \frac{1}{0.494579-\omega_{y}-\omega_{y}} d \omega_{y}=89.653979 \int_{0}^{t} d t .
\end{array}
$$

The left integral of equations is transformed and represented by the tabulated integral $\int d x /(a-x)=-\ln |a-x|+C$.
The right integral is simple, and integrals have the following solution:

$$
\begin{gathered}
-\ln \left|\frac{d \omega_{x}}{0.181102-\omega_{x}}-\omega_{x}\right|_{0}^{\omega_{x}}=\left.2058.7082 t\right|_{0} ^{t}, \\
-\ln \left|0.494579-\omega_{y}-\omega_{y}\right|_{0}^{\omega_{y}}=\left.89.653979 t\right|_{0} ^{t},
\end{gathered}
$$

that gave rise to the following:

$$
\begin{gathered}
1-\frac{\omega_{x}}{0.181102}=e^{-2058.7082 t}, \\
1-\frac{\omega_{y}}{0.494579-\omega_{y}}=e^{-89.653979 t} .
\end{gathered}
$$

The right side of Equations (32) and (32) has the small value of the high order that can be neglected. Solving these equations yields the angular velocity for the top around axis oy and $o x$ as the result of the action of the frictional torque.

$$
\begin{gathered}
\omega_{x}=0.181102 \mathrm{rad} / \mathrm{s}=10.376413^{\circ} / \mathrm{s}, \\
\omega_{y}=0.494579-\omega_{y} \mathrm{rad} / \mathrm{s}=28.337299^{\circ} / \mathrm{s} .
\end{gathered}
$$

Analysis of the angular velocities of the top around axes $o x$ and $o y$ demonstrates the turn down around axis $o y$ faster than around axis $o x$, and the rod contacts with the support surface. Following the action of the inertial forces on the Tippe top lifts up its center mass and disconnects the spherical part from the support surface. 
(2) The spinning Tippe top motions on the rod around vertical axes $o x$ and $o y$ (Figure 2). The top parameters defined above and in Table 2 are substituted into
Equations (21) and (22) that yield the following equations:

$$
\begin{gathered}
1.734 \times 10^{-5} \frac{d \omega_{x}}{d t}=\left[0.42 \times 0.04 \cos \left(-53.130102^{\circ}\right) \times \sin 53.130102^{\circ}+\frac{0.04 \cos \left(-53.130102^{\circ}\right)}{(3 / 8)(\pi-2)^{2} \pi+1}\right] \times 0.07 \times 9.81-\left[\frac{3}{8}(\pi-2)^{2} \pi+\frac{3}{8}(\pi-2)+\frac{6(\pi-2)^{2} \pi+3(\pi-2)+8}{8-3(\pi-2)}\right] \times 1.734 \times 10^{-5} \times 2000 \times\left(\frac{2 \pi}{60}\right) \times \omega_{x}, \\
1.734 \times 10^{-5} \frac{d \omega_{y}}{d t}=\left\{\frac{\left[(3 / 8)(\pi-2)^{2} \pi+1\right]\left(0.42 \times \sin -53.130102^{\circ}+1\right) \times 0.04 \cos \left(-53.130102^{\circ}\right)}{[(\pi-2) \pi+(8 /(3(\pi-2)))]\left[(3 / 8)(\pi-2)^{2} \pi+(3 / 8)(\pi-2)+\left(\left(6(\pi-2)^{2} \pi+3(\pi-2)+8\right) /(8-3(\pi-2))\right)\right]}\right\} \times 0.07 \times 9.81-\frac{3}{8}(\pi-2) \times 1.734 \times 10^{-5} \times 2000 \times\left(\frac{2 \pi}{60}\right) \times \omega_{y} .
\end{gathered}
$$

Following simplification and transformation is the same as presented above, and all comments for mathematical processing are omitted:

$$
\begin{gathered}
1.734 \times 10^{-5} \frac{d \omega_{x}}{d t}=0.012037-0.035698 \omega_{x}, \\
1.734 \times 10^{-5} \frac{d \omega_{y}}{d t}=9.589250 \times 10^{-4}-0.0015546 \omega_{y}, \\
\frac{d \omega_{x}}{0.337184-\omega_{x}}=2058.7082 d t \\
\frac{d \omega_{y}}{0.616830-\omega_{y}}=89.653979 d t \\
\int_{0}^{\omega_{x}} \frac{1}{0.337184-\omega_{x}} d \omega_{x}=2058.7082 \int_{0}^{t} d t \\
\int_{0}^{\omega_{y}} \frac{1}{0.616830-\omega_{y}} d \omega_{y}=89.653979 \int_{0}^{t} d t, \\
-\ln \left|0.337184-\omega_{x}\right|_{0}^{\omega_{x}}=\left.2058.7082 t\right|_{0} ^{t}, \\
-\ln \left|0.616830-\omega_{y}\right|_{0}^{\omega_{y}}=\left.89.653979 t\right|_{0} ^{t}, \\
1-\frac{\omega_{x}}{0.337184}=e^{-2058.7082 t}, \\
1-\frac{\omega_{y}}{0.616830}=e^{-89.653979 t}, \\
\omega_{x}=0.337184 \mathrm{rad} / \mathrm{s}=19.319220^{\circ} / \mathrm{s}, \\
\omega_{y}=0.616830 \mathrm{rad} / \mathrm{s}=35.341755^{\circ} / \mathrm{s} .
\end{gathered}
$$

Equations (34), (35), (47), and (48) demonstrate the top rotates faster around axis oy. At the time of the contact and disconnecting of the rod with the support surface, the angular velocities are different that are $\omega_{y}=28.337299^{\circ} / s$ (Equation (35)) and $\omega_{y}=35.341755^{\circ} / \mathrm{s}$ (Equation (48)), respectively. This difference is explained by the change in the radius of the frictional torque that is $l=R+r \sin \beta$ and $l=(R+k+r) \sin \beta$ and by the removing of the rolling frictional torque, respectively. The increase of the radius increases the value of the frictional torque and hence increases the angular velocities of the top rotation around axes. The Tippe top turns in the counterclockwise direction around axes ox and oy (Equations (34), (35), (47), and (48)). The action of the inertial forces on the spinning Tippe top that has the only contact of the rod with the support surface lifts up its center mass and the top goes to the vertical. Following spinning of the Tippe top on the rod is the same as for ordinary top.

The time of the spinning Tippe top inversion is computed for two conditions, i.e., for the turn from vertical to the angle of the contact of the rod with the support surface and for the following turn on the rod to vertical. Equations (35) and (48) demonstrate the angular velocities of the Tippe top rotation are changed by the cosine law. For solution is formulated the functional dependency of the variable angular velocity from the angle of the to turn that is as follows: $\omega_{i}$ $=a_{i} \cos \beta$ where $a_{i}$ is coefficient that defined for the two conditions, i.e., for the contact of the rod of the support surface and after disconnecting the spherical surface of the top of the support surface.

$28.337299^{\circ} / s=a_{c} \cos (-53.130102) \quad$ where $a_{c}=$ $47.228831^{\circ} / s$ and for $90^{\circ} \omega_{x}=0$.

$35.341755^{\circ} / s=a_{d} \cos (-53.130102) \quad$ where $a_{d}=$ $58.902925^{\circ} / s$ and for $\left(-90^{\circ}\right) \omega_{x}=0$. The time of the turn of the top on the angle $\beta$ can be computed by the equation: $t$ $=\beta_{i} / \omega_{i}$, where $\beta_{i}$ is the fixed angle of the top turn down that is $\beta_{d}=143.130102^{\circ}$ and for the turn up $\beta_{u}=90^{\circ}-$ $53.130102^{\circ}=36.869898^{\circ}$. The first derivative of variable angular velocity yields the following:

$$
d \omega_{i}=-a_{i} \sin \beta d \beta
$$

Integral presentation of Equation (49) brings the following:

$$
\int_{0}^{\omega_{i}} d \omega_{i}=-a_{i} \int_{s}^{e} \sin \beta d \beta
$$

where the limits of the integral $s$ are the start angle and $e$ is the end angle of the turn of the top.

Solution of the integral Equation (50) is presented by the following expression:

$$
\left.\omega_{i}\right|_{s} ^{e}=\left.a_{i} \cos \beta\right|_{s} ^{e},
$$

where all parameters are as specified above 
Substituting the initial data presented above for the top turn down and up, and solution yields the following results:

$$
\left.\omega_{d}\right|_{0} ^{\omega_{d}}=\left.47.228831^{\circ} \cos \beta\right|_{90 \circ} ^{-53.130102 \circ} \quad \text { and }\left.\omega_{u}\right|_{0} ^{\omega_{d}}=
$$
$\left.58.902925^{\circ} \cos \beta\right|_{-53.130102} ^{-90 \circ}$ that gave rise to the integrated angular velocities:

$$
\omega_{d}=47.228831\left[\cos \left(-53.130102^{\circ}\right)-\cos 90^{\circ}\right]=28.337299^{\circ} / s \text {, }
$$

$$
\omega_{u}=58.902925^{\circ}\left[\cos \left(-90^{\circ}\right)-\cos \left(-53.130102^{\circ}\right)\right]=35.341755^{\circ} / s .
$$

The time of the to turn down and up is defined by the expression $t_{i}=\beta_{i} / \omega_{i}$.

$$
\begin{aligned}
t_{d} & =\frac{143.130102}{28.337299}=5.050 \mathrm{~s}, \\
t_{u} & =\frac{36.869898}{35.341755}=1.043 \mathrm{~s} .
\end{aligned}
$$

The total time of the Tippe top inversion is as follows:

$$
t=t_{d}+t_{u}=5.050+1.043=6.093 s .
$$

\section{Results and Discussion}

The mathematical model for the motions of the Tippe top is derived on the principles of the theory of gyroscopic effects for rotating objects [17]. The inversion process of the Tippe top is the result of the action of the system of interrelated inertial torques generated by the rotating mass. The intuitive statement of the researchers that that frictional force acting on the Tippe top resulting in its inversion is not correct [516]. The frictional force acting on the spinning top activates the inertial torques of the Tippe top. The Tippe top manifests two precession rotations around two axes which are manifested by its inversion. The mathematical models for the Tippe top motions were used for computing the time of inversion from vertical to the upside-down vertical location. Practical observation of the similar Tippe top designs confirms the obtained results. The time of inversion will be less for the inclined axis of the Tippe top at the starting condition and for the rational size of the center mass displacement. This solution is analytical validation of the physics of the spinning Tippe top inversion.

\section{Conclusion}

Mathematical modeling of gyroscopic effects was problematic until the latest analytical solutions for the inertial torques acting on the spinning object of different designs. These inertial torques and motions of the spinning objects, which are known as gyroscopic effects, are the manifestations of the causal dependencies of the inertial torques and kinetic energies. These properties of the spinning objects constitute the fundamental principles of gyroscope theory. The application of these principles is demonstrated on the Tippe top inversion which presents the action of its precession torques. All unexplainable gyroscopic motions are solved by the contemporary principles of classical mechanics, and the Tippe top inversion is a good example of this one.

\section{Nomenclature}

$\begin{array}{ll}a, b, c, d, h, k, r, l_{c}, R: \begin{array}{l}\text { Geometrical sizes of the top } \\ m:\end{array} & \text { components } \\ g: & \text { Mass of the top } \\ f: & \text { Gravity acceleration } \\ f_{r}: & \text { Kinetic coefficient of sliding friction } \\ i: & \text { Coefficient of rolling friction } \\ J: & \text { Index for axis ox or oy } \\ J_{i}: & \text { Mass moment of inertia of the top } \\ T_{i}: & \text { Mass moment of inertia of the top } \\ T_{a m . i}: & \text { around axis } i \\ T_{c t i}, T_{c r . i}, T_{\mathrm{in} \cdot i}: & \text { Torque } i \\ & \text { Torque of the change in the angular } \\ T_{p . i}: & \text { momentum acting around axis } i \\ T_{r . i}: & \text { Torque generated by centrifugal, Cor- } \\ t: & \text { respectively, and acting around axis } i \\ \beta: & \text { Precession torque acting around axis } i \\ \gamma: & \text { Resistance torque acting around axis } i \\ \omega_{i}: & \text { Time } \\ \omega_{i}: & \text { Angle of inclination of the rotor's axle } \\ & \text { Angle of minor inclination of the }\end{array}$

\section{Data Availability}

The equation of inertial torque data used to support the findings of this study has been deposited in the journal AIP Advances that is in the process.

\section{Conflicts of Interest}

The authors declare that they have no conflicts of interest.

\section{Acknowledgments}

The work was supported by the Kyrgyz State Technical University after I. Razzakov.

\section{References}

[1] J. R. Taylor, Classical Mechanics, University Science Books, Mill Valley, CA. USA, 2005.

[2] R. C. Hibbeler and K. B. Yap, Mechanics for Engineers - Statics and Dynamics, Prentice Hall, Pearson, Singapore, 13th edition, 2013.

[3] D. R. Gregory, Classical Mechanics, Cambridge University Press, New York, 2006.

[4] N. M. Bou-Rabee, J. E. Marsden, and L. A. Romero, "Tippe top inversion as a dissipation-induced instability," SIAM Journal on Applied Dynamical Systems, vol. 3, no. 3, pp. 352-377, 2004. 
[5] R. Cross, "Dynamics of a spherical tippe top," European Journal of Physics, vol. 39, no. 3, article 035001, 2018.

[6] M. C. Ciocci, B. Malengier, B. Langerock, and B. Grimonprez, "Towards a prototype of a spherical tippe top," Journal of Applied Mathematics, vol. 2012, 34 pages, 2012.

[7] M. C. Ciocci and B. Langerock, "Dynamics of the tippe top via Routhian reduction," Regular and Chaotic Dynamics, vol. 12, no. 6, pp. 602-614, 2007.

[8] N. Rutstam, "Tippe top equations and equations for the related mechanical systems," SIGMA. Symmetry, Integrability and Geometry: Methods and Applications, vol. 8, article 019, 2012.

[9] S. T. Glad, D. Petersson, and S. Rauch-Wojciechowski, "Phase space of rolling solutions of the tippe top," SIGMA. Symmetry, Integrability and Geometry: Methods and Applications, vol. 3, article 041, 2007.

[10] H. K. Moffatt and Y. Shimomura, "Classical dynamics: spinning eggs - a paradox resolved," Nature, vol. 416, no. 6879, pp. 385-386, 2002.

[11] H. K. Moffatt, Y. Shimomura, and M. Branicki, "Dynamics of axisymmetric body spinning on a horizontal surface. I. Stability and the gyroscopic approximation," Proceedings of the Royal Society of London. Series A: Mathematical, Physical and Engineering Sciences, vol. 460, pp. 3643-3672, 2004.

[12] S. Rauch-Wojciechowski, "What does it mean to explain the rising of the tippe top?," Regular and Chaotic Dynamics, vol. 13, no. 4, pp. 316-331, 2008.

[13] N. Rutstam, "Study of equations for tippe top and related rigid bodies," in Linköping Studies in Science and Technology, theses no. 1106, Matematiska Institutionen, Linköpings Universitet, 2010, http://urn.kb.se/resolve?urn=urn:nbn:se:liu:diva-60835.

[14] T. Ueda, K. Sasaki, and S. Watanabe, "Motion of the tippe top: gyroscopic balance condition and stability," SIAM Journal on Applied Dynamical Systems, vol. 4, no. 4, pp. 1159-1194, 2005.

[15] S. Dastjerdi, B. Akgöz, and O. Civalek, "On the effect of viscoelasticity on behavior of gyroscopes," International Journal of Engineering Science, vol. 149, p. 103236, 2020.

[16] C. Demir and O. Civalek, "On the analysis of microbeams," International Journal of Engineering Science, vol. 121, pp. 1433, 2017.

[17] R. Usubamatov, Theory of Gyroscopic Effects for Rotating Objects, Springer, Singapore, 2020.

[18] R. Usubamatov, "Inertial torques acting on a spinning sphere," AIP Advances, 2021.

[19] http://www.EngineeringToolbox.com/. 